\title{
Changes in Vestibular Symptoms and Function After Cochlear Implantation: Relevant Factors and Correlations With Residual Hearing
}

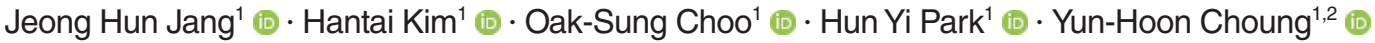 \\ ${ }^{1}$ Department of Otorhinolaryngology, Ajou University School of Medicine, Suwon; ${ }^{2}$ BK21 Plus Research Center for Biomedical Sciences, \\ Ajou University Graduate School of Medicine, Suwon, Korea
}

Objectives. The aim of this study was to evaluate vestibular function loss after cochlear implantation (CI) and the relationship between vestibular function and hearing changes.

Methods. Seventy-five patients with CI were enrolled and divided into those with normal preoperative caloric function (group I) and those with a normal preoperative waveform in cervical vestibular evoked myogenic potential (c-VEMP) testing (group II). The relationship between hearing and changes in the vestibular system was analyzed preoperatively and at 3 and 6 months postoperatively.

Results. In group I, unilateral weakness on the implanted side was detected in five (7.7\%) and eight (12.3\%) patients at 3 and 6 months post-CI, respectively. By 3 months post-CI, the total slow-phase velocity (SPV; warm and cold stimulations) was significantly different between the implanted and non-implanted sides $(P=0.011)$, and the shift in total SPV from pre- to post-CI was significantly correlated with the average hearing threshold at 6 months post-CI. In group II, an abnormal c-VEMP was detected on the implanted side in six patients $(16.2 \%)$ at 3 months post-CI, and in six patients $(16.2 \%)$ at 6 months post-CI. Significant changes were noticed in the P1 and N1 amplitude at 3 months postCI $(P=0.027$ and $P=0.019$, respectively).

Conclusion. Vestibular function and residual hearing function should be afforded equal and simultaneous consideration in terms of preservation.

Keywords. Cochlear Implantation; Vestibular Function Tests; Vertigo; Caloric Tests; Vestibular Evoked Myogenic Potential

\section{INTRODUCTION}

It is widely acknowledged that electrode insertion during cochlear implantation (CI) may result in iatrogenic damage to the inner ear. Because residual hearing is often lost during the $\mathrm{CI}$ procedure, post-CI preservation of residual hearing has been a major focus of research. The preservation rate ranges between $21.4 \%$ and $56.5 \%[1,2]$. Recent studies have proposed a variety

- Received November 12, 2019

Revised February 9, 2020

Accepted March 5, 2020

- Corresponding author: Yun-Hoon Choung

Department of Otorhinolaryngology, Ajou University School of Medicine,

206 World cup-ro, Yeongtong-gu, Suwon 16499, Korea

Tel: +82-31-219-5263, Fax: +82-31-219-5264

E-mail: yhc@ajou.ac.kr of techniques for improving the rate of residual hearing preservation, including "soft surgery" $[3,4]$.

The cochlea and vestibule are subsystems of the labyrinth. Although the surgical procedure for $\mathrm{CI}$ does not directly involve the vestibular structure, insertion of electrodes into the scala tympani may affect the vestibular structure in various ways, depending on the anatomical and physiological relationships at play. Several scenarios have been proposed to account for vestibular damage ensuing from $\mathrm{CI}$, including direct trauma from electrode insertion, intraoperative perilymph loss, foreign body reaction or labyrinthitis, endolymphatic hydrops, and electrical vestibular stimulation. The prevalence of subjective dizziness post-CI is estimated to be $9.3 \%$ [5] and that of vestibular deficit after $\mathrm{CI}$ ranges from $39 \%$ to $74 \%$ [6-9]. The saccule is the part of the vestibular system that is most commonly damaged during

Copyright $@ 2021$ by Korean Society of Otorhinolaryngology-Head and Neck Surgery.

This is an open-access article distributed under the terms of the Creative Commons Attribution Non-Commercial License (https://creativecommons.org/licenses/by-nc/4.0)

which permits unrestricted non-commercial use, distribution, and reproduction in any medium, provided the original work is properly cited. 
the CI procedure, followed by the utricle and semicircular canals [10]. Anatomically, the ductus reuniens connects the endolymphatic space between the cochlear duct and the saccule. If obstruction of the ductus reuniens or cochlear duct occurs as a result of implantation, the endolymphatic flow will be blocked, and consequently, the saccule will collapse [11].

Several studies have analyzed the factors associated with CI that influence hearing preservation, including insertion depth angle, steroid administration, electrode length, surgical approach, and electrode type. In recent years, the contribution of these factors to the preservation of vestibular function has been the subject of extensive and compelling research, but the follow-up periods in these studies were relatively short and decisive conclusions remain elusive $[9,12]$. In addition, few reports have examined the relationship between residual hearing and vestibular function after CI, although Nordfalk et al. [13] reported that the loss of caloric response was not associated with the loss of residual hearing. We enrolled subjects with normal vestibular function and analyzed serial changes in their respective vestibular symptoms and function. In addition, we evaluated the correlations of vestibular change with other relevant aspects of the CI technique, as well as residual hearing.

\section{MATERIALS AND METHODS}

This study was approved by the Institutional Review Board of the Ajou University School of Medicine (IRB No. AJIRB-MEDMDB-18-161). The requirement for informed consent was waived.

Of 401 patients who underwent CI between February 2002 and December 2017 at the CI Center of the Department of Otolaryngology, Ajou University Hospital, 75 patients for whom the results of hearing and vestibular function tests were available were included in this study. A total of 75 patients were categorized into two conditions, as appropriate, for analyses. Group I $(n=65)$ comprised patients with preoperative normal caloric function; group II ( $\mathrm{n}=37)$ included patients with a preoperative-

\section{H I G G H L I G G H T S}

- Caloric testing showed unilateral weakness on the implanted side in five patients $(7.7 \%)$ and eight patients $(12.3 \%)$ at 3 and 6 months after cochlear implantation (CI), respectively.

- An abnormal cervical vestibular evoked myogenic potential was found on the implanted side in six patients $(16.2 \%)$ and six patients (16.2\%) at 3 and 6 months post-CI, respectively.

- Vestibular parameters, such as the total slow-phase velocity and amplitudes of P1 and N1, significantly decreased on the implanted side at 3 months post-CI.

- Vestibular function and residual hearing function should be afforded equal and simultaneous consideration in terms of preservation.
401 Patients with Cl (Feb 2002-Dec 2017)

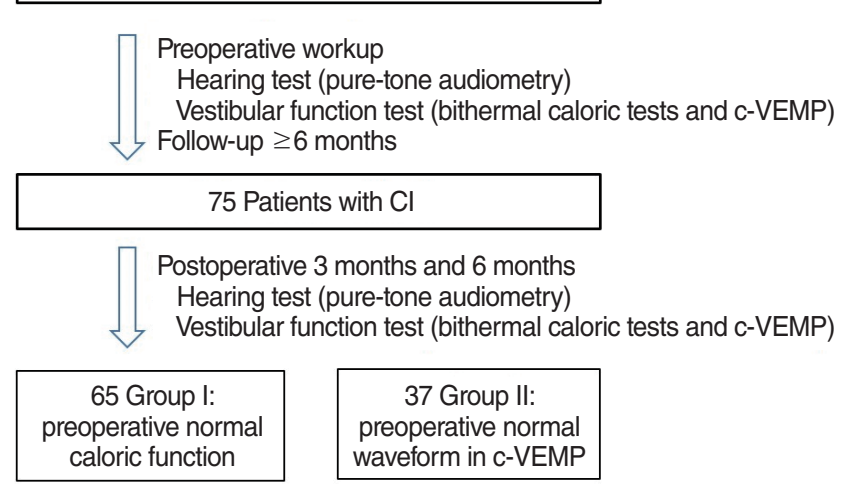

Fig. 1. Flowchart of this study. $\mathrm{Cl}$, cochlear implantation; c-VEMP, cervical vestibular evoked myogenic potential.

ly normal waveform in cervical vestibular evoked myogenic potential (c-VEMP) testing. Demographic and clinical data, including sex, age, the side on which surgery had been performed, and diagnosis, were retrospectively reviewed (Fig. 1).

The patient's subjective dizziness was clinically assessed through face-to-face questionnaire preoperatively and postoperatively. Questionnaire was designed to collect data on the type, duration, and intensity of dizziness. The vestibular function test battery was performed in the following sequence: bithermal caloric tests and c-VEMP tests were performed preoperatively, and again at 3 months, and 6 months postoperatively. Hearing status was also evaluated in accordance with the same schedule, using pure-tone audiometry. The change of residual hearing and vestibular function was compared.

\section{Bithermal caloric tests}

Bithermal caloric tests evaluate the degree to which responses in the lateral semicircular canals of each ear are symmetric, by performing two separate stimulations, at $44^{\circ} \mathrm{C}$ and $30^{\circ} \mathrm{C}$, using a water caloric stimulator (NCI-480; ICS). Unilateral weakness (UW) and angular velocity of the slow phase for each stimulation, on both sides, were compared before and after CI. Responses were recorded using a three-dimensional videonystagmography mono-oculography system (SLVNG; SLMED, Seoul, Korea). The caloric test was performed with the patient in a reclining position, with the head turned $30^{\circ}$ so that the lateral canal was horizontal. Water was introduced into the ear canal on one side and stopped after 30 seconds, at which point the patient was distracted with quiz questions. Nystagmus usually builds in intensity over around 30 seconds, then gradually decreases over approximately 2 minutes. Each test was performed following a rest of at least 5 minutes; the procedure was repeated for both ears with warm stimulation applied first. Based on the canal paresis values of the bithermal caloric tests for 30 healthy volunteers, we derived $27 \%$, the criteria for the pathologic UW of the equipment in our hospital. A value $>27 \%$ was deemed to indi- 
cate pathological UW.

\section{c-VEMP}

The c-VEMP test determines whether the saccule, the otolith, and the inferior vestibular nerve and central connections are intact and working normally. In this study, VEMP analyses were conducted using an evoked potential system (Navigator PRO; Bio-logic Systems Corp., Mundelein, IL, USA). Patients were examined in a supine position, with the head turned in the opposite direction to that of the stimulated ear. The tests were performed using air-conduction tone bursts. Responses were recorded using an active electrode, placed on the mid-point of each sternocleidomastoid muscle, while a reference electrode was placed on the sternum and the ground electrode was placed in the middle of the forehead. The acoustic stimulus comprised a short tone burst, $500 \mathrm{~Hz}$ at $90 \mathrm{~dB} \mathrm{HL}$, delivered through insert earphones. The duration of the analyses were $100 \mathrm{~ms}$, and the electromyographic signal was band-pass filtered from $500 \mathrm{~Hz}$ to $2 \mathrm{kHz}$. Every set of 200 stimuli was averaged and repeated three times to verify the reproducibility of the response. VEMP asymmetry (VA) was defined as a difference in threshold and/or amplitude between both ears. Abnormal c-VEMP was categorized as the combination of VA or absence of response.

\section{Pure-tone audiometry}

Hearing threshold was calculated pre- and postoperatively by averaging low frequencies $(250,500$, and 1,000 Hz) and only the patients with functional residual hearing were included in the analysis. Postoperative tests were performed using headphones to analyze the residual audiometric threshold.

\section{Statistical analysis}

All data were compared before and after CI, with parametric tests using IBM SPSS ver. 21 (IBM Corp., Armonk, NY, USA). Factors related with subjective dizziness were analyzed using chi-square test. Caloric response and c-VEMP were compared among electrode type, approach, and steroid administration using $t$-test. The correlation analysis was performed if the change of hearing threshold would be related with the change of caloric response and c-VEMP. In all analyses, a value of $P<0.05$ was taken to indicate statistical significance.

\section{RESULTS}

The study cohort comprised 30 males and 45 females with a mean age of $37.4 \pm 23.3$ years (range, $2.9-79.0$ years) at the time of CI. In total, 43 ears $(57.3 \%)$ were fitted with cochlear devices (Cochlear Ltd., Sydney, Australia): nine ears (12.0\%) were fitted with Advance Bionics devices (Advanced Bionics, Sylmar, CA, USA); 21 ears (28.0\%) were fitted with MED-EL devices (MEDEL, Innsbruck, Austria); and two ears (2.7\%) were fitted with
Table 1. Demographic characteristics of patients $(n=75)$

\begin{tabular}{lcc}
\hline Characteristics & Group I $(\mathrm{n}=65)$ & Group II $(\mathrm{n}=37)$ \\
\hline Male:female & $24: 41$ & $15: 22$ \\
Right:left:both & $34: 26: 5$ & $15: 19: 3$ \\
Age at Cl (yr), mean \pm SD (range) & $38.7 \pm 23.6$ & $32.8 \pm 20.7$ \\
& $(2.9-79.0)$ & $(9.0-74.2)$ \\
& $41: 8: 15: 2$ & $21: 1: 14: 1$ \\
Device (Cochlear:Advanced & & \\
$\quad$ Bionics:MED-EL:Oticon Medical) & $12: 39: 14$ & $5: 18: 14$ \\
Electrode type & & \\
$\quad$ (precurved:straight:flexible) & $16: 47$ & $4: 29$ \\
Approach (cochleostomy:round window) & $48: 17$ & $34: 3$ \\
\hline Steroid administration (+:-) &
\end{tabular}

Group I, preoperative normal bithermal caloric function; Group II, preoperative normal c-VEMP waveform; Preoperative normal bithermal caloric function and c-VEMP waveform: $n=27$. Group I and Group II are not mutually exclusive.

$\mathrm{Cl}$, cochlear implantation; SD, standard deviation; c-VEMP, cervical vestibular evoked myogenic potential.

Oticon Medical Neurelec CI systems (Oticon Medical, Vallauris, France). Regarding the type of electrodes, precurved electrodes (Cochlear Ltd.: 24RCA, 24RECA, and CI512), straight electrodes (Cochlear Ltd.: CI422; Advanced Bionics: HiFocus 1J; Oticon Medical: EVO), and flexible electrodes (MED-EL: Flex 24, 28, and soft) were inserted.

\section{Group I: preoperative normal bithermal caloric function}

Among the 75 patients, 65 showed normal bithermal caloric tests preoperatively (Table 1). UW was detected on the implanted side in five patients $(7.7 \%)$, and in a further eight patients $(12.3 \%)$ at 3 months and 6 months post-CI, respectively. The maximum slow-phase velocity (SPV) total (from warm and cold stimulation) of the implanted side was $48.4 \pm 26.4$ preoperatively, $35.3 \pm 19.6$ at 3 months post-CI, and $37.7 \pm 22.6$ at 6 months post-CI. The SPV totals of the implanted side and the non-implanted side were compared. While the SPV total (warm and cold stimulations) did not differ significantly between the implanted and non-implanted sides preoperatively $(P>0.05)$, a significant difference was found at 3 months post-CI $(P=0.011)$, but not at 6 months $(P=0.133)$. The shift in the SPV total (i.e., the disparity between the pre-CI and post-CI SPV totals) on the implanted side increased with age at the time of $\mathrm{CI}$ at 3 months post-CI ( $r=0.280, P=0.034)$, but not at 6 months post-CI.

Of the 19 patients who reported subjective dizziness preoperatively, eight reported the continuation of subjective dizziness at 3 months post-CI, and seven did so at 6 months post-CI. There was no correlation between persistent subjective dizziness postoperatively and UW in the caloric tests at 3 and 6 months postoperatively. Among the patients that did not report any dizziness preoperatively, postoperative dizziness newly developed in eight patients at 3 months post-CI and in five patients at 6 months post-CI. The SPV total was lower among patients with

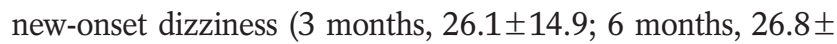



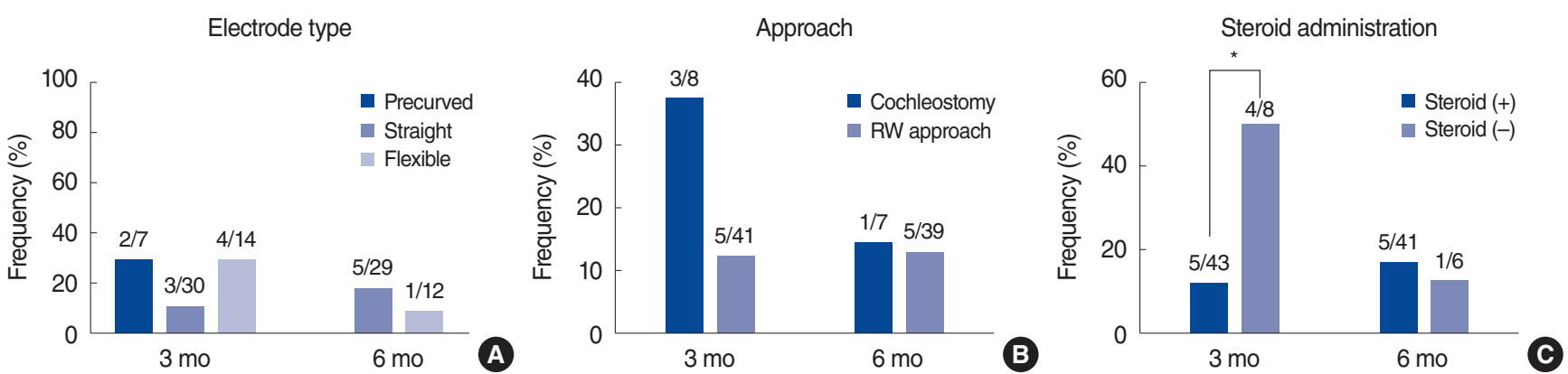

Fig. 2. The frequency of new-onset subjective dizziness according to electrode type (A), approach (B), and steroid administration (C). At 3 months post-cochlear implantation, subjective dizziness was somewhat more frequent in patients who underwent cochleostomy $(P=0.077)$, and significantly more frequent in patients who had not been administered steroids $(P=0.009)$. The statistical analysis was performed using the chi-square test. RW, round window. ${ }^{*} P<0.05$.
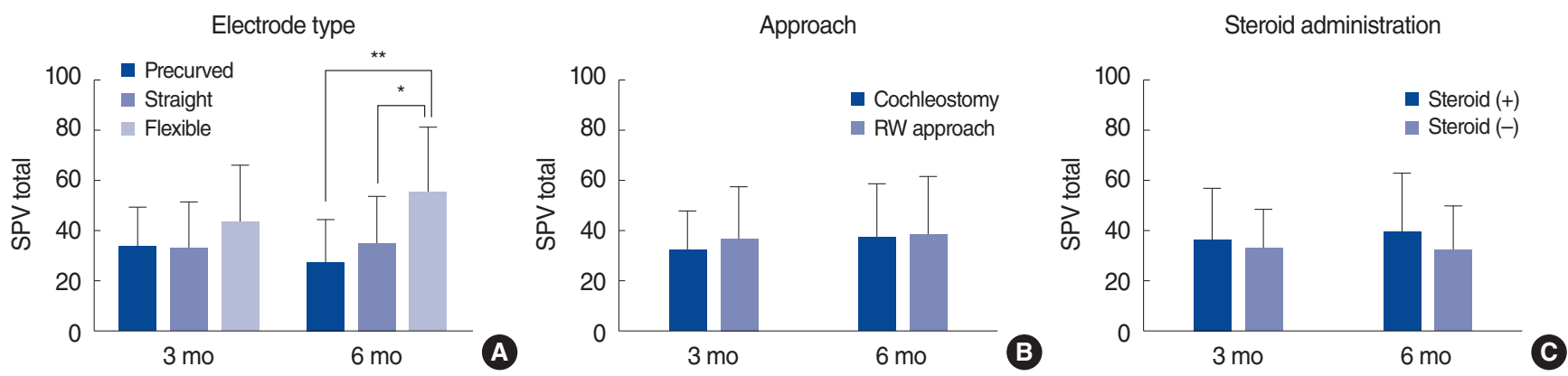

Fig. 3. Comparison of the maximum slow-phase velocity (SPV) total (warm and cold stimulations) according to the electrode type (A), approach (B), and steroid administration (C). The SPV total was greater in ears with cochlear implantation $(\mathrm{Cl})$ using a flexible electrode $(3$ months, 43.3 $\pm 23.0 ; 6$ months, 55.3 \pm 26.4 ) than in ears with $\mathrm{Cl}$ using a precurved electrode (3 months, 33.2 $\pm 16.7 ; 6$ months, 27.2 \pm 17.4$)$ or a straight electrode (3 months, 32.9 \pm 18.8 ; 6 months, 34.5 \pm 19.4$)$, and the difference was statistically significant at 6 months post-Cl $(P=0.007)$. The statistical analysis was performed using the $t$-test. RW, round window. ${ }^{\star} P<0.05,{ }^{\star \star} P<0.01$.

16.6) than among the others (3 months, $35.9 \pm 19.2 ; 6$ months, 40.3 \pm 24.6$)$, but this difference was not significant.

Electrode type, approach, and steroid administration were analyzed in relation to subjective dizziness. The schedule of oral steroid administration was a 10-day course of prednisolone (Solondo; Yuhan, Seoul, Korea) consisting of $60 \mathrm{mg} /$ day for 5 days, $40 \mathrm{mg} /$ day for 2 days, $20 \mathrm{mg} /$ day for 2 days, and $10 \mathrm{mg} /$ day for 1 day. $\mathrm{CI}$ was performed on the second day. At 3 months post-CI, subjective dizziness was somewhat more frequent among patients who had undergone cochleostomy $(P=0.077)$, and significantly more frequent among patients who had not been administered steroids $(P=0.009)$. However, no significance was observed at 6 months post-CI (Fig. 2). Postoperative UW was not associated with electrode type, approach, or steroid administration at 3 months and 6 months post-CI.The SPV total was greater in patients whose $\mathrm{CI}$ had been performed via the round window approach than in those whose $\mathrm{CI}$ was executed via cochleostomy, both at 3 months and 6 months post-CI, but the differences were not significant. The SPV total was also greater in instances of CI with associated steroid administration than in instances of $\mathrm{CI}$ without any steroid administration, both at 3 months and 6 months post-CI, but again the difference was not significant. However, the SPV total was greater among patients in whom $\mathrm{CI}$ was performed using a flexible electrode (3 months, $43.3 \pm 23.0$; 6 months, $55.3 \pm 26.4$ ) than among those in whom $\mathrm{CI}$ was performed using a precurved electrode ( 3 months, $33.2 \pm 16.7 ; 6$ months, $27.2 \pm$ 17.4) or straight electrode ( 3 months, $32.9 \pm 18.8$; 6 months, $34.5 \pm 19.4$ ), both at 3 months and 6 months post-CI, and in this case the difference was statistically significant at 6 months post$\mathrm{CI}(P=0.007)$ (Fig. 3).

Preoperative and postoperative hearing threshold data were available for 28 patients in group I. The threshold shift (the disparity between the pre-CI and post-CI thresholds) did not significantly differ between patients with UW and those with a normal caloric response at 3 months and 6 months post-CI. However, the shift in the SPV total (corrected for age at the time of $\mathrm{CI}$ ) was significantly correlated with the average hearing threshold shift at 6 months post-CI $(r=0.701, P=0.002)$ but not at 3 months post-CI (Fig. 4).

\section{Group II: preoperative normal waveform of c-VEMP}

Among the 75 patients, 37 patients showed normal c-VEMP data preoperatively (Table 1). An abnormal c-VEMP on the implanted side was detected in six of these patients $(16.2 \%)$ and 


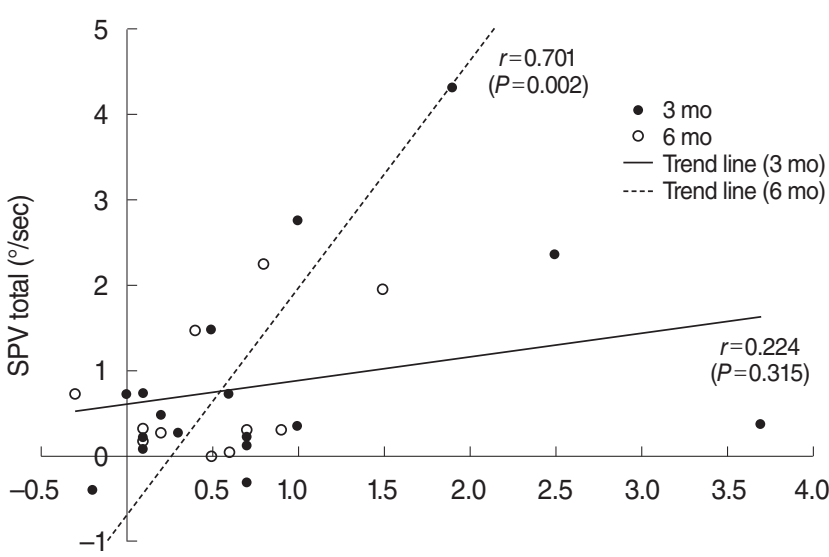

Average thresholds shift $(\mathrm{dB})$

Fig. 4. Correlation between the average hearing threshold shift and the shift of the maximum slow-phase velocity (SPV) total (difference between the preoperative SPV total and postoperative SPV total) in group I. The statistical analysis was performed using correlation analysis.

in a further six patients (20\%) at 3 months and 6 months post$\mathrm{CI}$, respectively. While the P1 latency, N1 latency, and interpeak latency were stationary irrespective of $\mathrm{CI}$, the $\mathrm{P} 1$ and $\mathrm{N} 1$ amplitude decreased following CI. Parameters were compared between the implanted and non-implanted sides. While all parameters were similar preoperatively $(P>0.05)$, the $\mathrm{P} 1$ amplitude and $\mathrm{N} 1$ amplitude significantly decreased at 3 months post-CI ( $P=0.027$ and $P=0.019$, respectively) but not at 6 months post$\mathrm{CI}$. For reference, there was no significant change in the c-VEMP data on the non-implanted side at 3 months and 6 months postCI $(P=0.109$ and $P=0.108$, respectively).

Of 13 patients who reported subjective dizziness preoperatively, five reported continued subjective dizziness at 3 months, and three did so at 6 months post-CI. Of the patients who did not suffer dizziness preoperatively, new-onset dizziness was reported postoperatively by five patients at 3 months and two patients at 6 months post-CI. No correlation was detected between subjective dizziness and abnormal changes in vestibular function tests.

Electrode type, approach, and steroid administration were analyzed in relation to subjective dizziness, and no relationship was discerned among them. Postoperative abnormal c-VEMP was also found to be unrelated to electrode type, approach, or steroid administration at either 3 months or 6 months post-CI. Pre- and postoperative hearing threshold data were available for 24 patients in group II. The threshold shift did not differ between the patients with an abnormal c-VEMP and those with a normal waveform at 3 months and 6 months post-CI.

\section{DISCUSSION}

Most previous studies have not specifically investigated preoperative vestibular function status. Under such conditions, hetero- geneous vestibular data are mixed, meaning that postoperative changes cannot be accurately interpreted. In the present study, patients preoperatively deemed to have normal caloric testing (group I) or c-VEMP results (group II) were analyzed. Generally, canal paresis is calculated using Jongkees' formula, and in our study, UW was defined as canal paresis in over $27 \%$ of cases. Because we intended to analyze changes in canal paresis among the entire cohort of patients, we included the SPV total on the implanted sides as a quantitative factor.

Vestibular function changes were evaluated prior to $\mathrm{CI}$ and at 6 months post-CI. The SPV total differed significantly between the implanted and non-implanted sides at 3 months post-CI, while no disparity was observed at 6 months post-CI in group I. Subjective dizziness was somewhat less frequently found depending on the approach, and was significantly less frequently in patients who received steroid administration, but only at 3 months post-CI in group I. In group II, while all parameters were similar preoperatively $(P>0.05)$, the $\mathrm{P} 1$ amplitude and N1 amplitude significantly decreased at 3 months post-CI $(P=0.027$ and $P=$ 0.019 , respectively). Xu et al. [14] reported that c-VEMP disappearance occurred significantly more often on the implanted side and that waveform parameters showed abnormal changes such as decreased amplitude, forward movement of P1 and N1, and shortened interpeak latency at 1 month after $\mathrm{CI}$, suggesting that c-VEMP waveforms could reflect the degree of damage to the saccule caused by $\mathrm{CI}$. The c-VEMP change in the present study showed a similar tendency at 3 months post-CI. Considering the small sample size, long-term changes in c-VEMP parameters should be explored through follow-up.

Electrode type, approach, and steroid administration may all contribute to postoperative changes in vestibular function. Todt et al. [9] compared VEMP response and electronystagmography and concluded that the round window approach was preferable for preventing vestibular injury. Frodlund et al. [15] reported that precurved electrodes were associated with a significant decrease in SPV and high prevalence of subjective vertigo. Enticott et al. [16]. performed a prospective clinical trial and reported that frequency of postoperative vertigo symptoms were significantly lower in patients who had been administered steroid treatment than the control group. In the present study, the frequency of new-onset subjective dizziness was related to the approach and steroid administration, but UW and an abnormal cVEMP were not related in group I. The SPV total was greater among patients who had been implanted with a flexible electrode than among those in whom precurved and straight electrodes had been used, and this difference was statistically significant at 6 months post-CI in group I.

We also investigated the relationship between residual hearing and changes in vestibular function. The shift in the SPV total was significantly correlated with the average hearing threshold only at 6 months post-CI in group I. This finding indicates that the first 6 months post-implantation may be critical for preserv- 
ing cochleovestibular system function. It is acknowledged that vestibular function, unlike residual hearing, may be recoverable through central compensation. As such, differences in vestibular function change at 6 months post-CI may be attributable to delayed central compensation. However, asymmetries in caloric function from a stable vestibular lesion could be quite stable long-term, even with central compensation.

Santa Maria et al. [1] reported a linear downward trend in hearing preservation over time. In that study, changes in hearing preservation were particularly significant from 0 to 3 and 0 to 6 months. Vestibular function in our study similarly showed a linear downward tendency over time, and we observed a correlation with residual hearing at 6 months post-CI in group I. We also examined the data from group I to identify characteristics that might predict which patients would suffer UW at 6 months post-CI, but no significant factors were identified (data not shown). A further study based on a large sample is required.

In our study, subjective dizziness was reported by nine patients at 3 months post-CI and six patients at 6 months post-CI in group I. No correlation was found between UW and/or an abnormal c-VEMP and subjective dizziness. There are various causes of subjective dizziness. Rah et al. [17] itemized the etiologies of postoperative dizziness as follows: transient vestibular paresis, benign paroxysmal postural vertigo, endolymphatic hydrops, vestibular migraine, and bilateral hypofunction. However, bithermal caloric tests evaluate the horizontal semicircular canal, while c-VEMP assesses saccular response, and as such, these tests cannot detect subjective dizziness. If the number of samples is sufficient, the SPV total would be expected to be significantly lower in among patients with new-onset dizziness than among others.

Table 2 presents the prevalence of vestibular hypofunction as reported in previous studies. The prevalence rates of UW $(12.3 \%)$ and abnormal c-VEMP $(20 \%)$ in this study are lower than the mean rates of UW (31.4\%) and abnormal c-VEMP $(54.3 \%)$ reported in previous studies. This discrepancy might reflect several factors, such as the duration of follow-up, popularization of the soft surgery technique, and perioperative steroid use. Although the extent varies across studies, we may surmise that deterioration of vestibular function is a risk associated with CI. The incidence of abnormal c-VEMP $(20 \%)$ was higher that of UW $(12.3 \%)$ in the present study, which is a similar finding to those of previous studies $[9,12,18-20]$. It is known that the utricle is the sensor that is most frequently damaged after CI [10]. If the ductus reuniens or cochlear duct is obstructed by electrode insertion, endolymph flow could be blocked and the saccule could collapse.

The audiological criteria for $\mathrm{CI}$ in the National Health Insurance in Korea are as follows: more than $90 \mathrm{~dB}$ of hearing loss at an age $\leq 24$ months (auditory brainstem response threshold), and more than $70 \mathrm{~dB}$ of hearing loss at an age $>24$ months (pure-tone average frequencies of 500, 1,000, 2,000, and 4,000 Hz). Since
Table 2. Overview of clinical studies on vestibular function before and after $\mathrm{Cl}$

\begin{tabular}{|c|c|c|c|}
\hline \multirow[t]{2}{*}{ Study } & \multicolumn{2}{|c|}{$\begin{array}{l}\text { Postoperative abnormal rate of patients } \\
\text { among normal preoperative vestibular } \\
\text { function }\end{array}$} & \multirow{2}{*}{$\begin{array}{l}\text { Mean } \\
\text { follow-up } \\
\quad(\mathrm{mo})\end{array}$} \\
\hline & Caloric test (\%) & c-VEMP (\%) & \\
\hline Ito [7] & 33 & & 1 \\
\hline Rossi et al. [21] & 3.1 & 6 & \\
\hline Todt et al. [9] & 19.4 & 21 & $1.5-2$ \\
\hline Basta et al. [18] & 5.6 & 55.6 & 1.5 \\
\hline Kluenter et al. [22] & 5.6 & & 1.5 \\
\hline Krause et al. [23] & 32 & & $1-1.5$ \\
\hline Katsiari et al. [19] & 60 & 60 & 1 \\
\hline Robard et al. [24] & 48.3 & 59 & 5 \\
\hline Louza et al. [25] & 83 & 62 & $1-1.5$ \\
\hline Nordfalk et al. [12] & 38.1 & 46.2 & $1.5-2$ \\
\hline Meli et al. [20] & 17.6 & 76.5 & 2 \\
\hline This study & 12.3 & 20 & 6 \\
\hline
\end{tabular}

$\mathrm{Cl}$, cochlear implantation; c-VEMP, cervical vestibular evoked myogenic potential.

the residual hearing of high frequencies of most $\mathrm{CI}$ candidates is too poor to be considered functional, the average values for low frequencies $(125,250$, and $500 \mathrm{~Hz})$ were used for analysis.

There are several limitations of this study that should be considered. Vestibular function data such as the SPV total and cVEMP are known to have high test-retest variability. Further analysis, including a quantitative study, is undergoing. Mastoidectomy and thinning of the posterior canal wall could affect caloric function. Since the caloric test and c-VEMP are primary measurement tools, additional data using the rotational chair test and/or video head impulse test would be needed.

The frequency of post-CI vestibular dysfunction was $12.3 \%$ for UW and $20 \%$ for an abnormal c-VEMP. The influence of CI on vestibular function was deemed to be potentially critical up to 3 months post-implantation, after which these effects may be minimal. The extent to which residual hearing was preserved was only demonstrably correlated with vestibular dysfunction at 6 months post-CI.Vestibular function and residual hearing should, therefore, be afforded equal and simultaneous consideration in terms of their preservation from the earliest stages of treatment.

\section{CONFLICT OF INTEREST}

No potential conflict of interest relevant to this article was reported.

\section{ACKNOWLEDGMENTS}

This research was supported by the Basic Science Research Program through the National Research Foundation of Korea fund- 
ed by the Ministry of Science, ICT \& Future Planning (NRF2018R1A1A1A05023057).

\section{ORCID}

$\begin{array}{ll}\text { Jeong Hun Jang } & \text { https://orcid.org/0000-0003-1707-5714 } \\ \text { Hantai Kim } & \text { https://orcid.org/0000-0002-5020-7814 } \\ \text { Oak-Sung Choo } & \text { https://orcid.org/0000-0002-1633-8546 } \\ \text { Hun Yi Park } & \text { https://orcid.org/0000-0003-2467-3114 } \\ \text { Yun-Hoon Choung } & \text { https://orcid.org/0000-0002-0786-1781 }\end{array}$

\section{AUTHOR CONTRIBUTIONS}

Conceptualization: YHC. Data curation: JHJ. Formal analysis: JHJ, HK. Methodology: HYP, OSC. Project administration: YHC. Visualization: OSC. Writing-original draft: JHJ. Writing-review \& editing: $\mathrm{YHC}, \mathrm{OSC}$.

\section{REFERENCES}

1. Santa Maria PL, Domville-Lewis C, Sucher CM, Chester-Browne R, Atlas MD. Hearing preservation surgery for cochlear implantation: hearing and quality of life after 2 years. Otol Neurotol. 2013 Apr; 34(3):526-31.

2. GstoettnerWK, Helbig S, Maier N, Kiefer J, RadeloffA, Adunka OF. Ipsilateral electric acoustic stimulation of the auditory system: results of long-term hearing preservation. Audiol Neurootol. 2006;11 Suppl 1:49-56.

3. Eshraghi AA,Ahmed J, Krysiak E, Ila K,Ashman P,Telischi FF, et al. Clinical, surgical, and electrical factors impacting residual hearing in cochlear implant surgery. Acta Otolaryngol. 2017 Apr;137(4):384-8.

4. Rajan GP, Kuthubutheen J, Hedne N, Krishnaswamy J. The role of preoperative, intratympanic glucocorticoids for hearing preservation in cochlear implantation: a prospective clinical study. Laryngoscope. 2012 Jan;122(1):190-5.

5. HanselT, Gauger U, Bernhard N, Behzadi N, Romo Ventura ME, Hofmann V, et al. Meta-analysis of subjective complaints of vertigo and vestibular tests after cochlear implantation. Laryngoscope. 2018 Sep;128(9):2110-23.

6. Fina M, Skinner M, Goebel JA, Piccirillo JF, Neely JG, Black O. Vestibular dysfunction after cochlear implantation. Otol Neurotol. 2003 Mar;24(2):234-42.

7. Ito J. Influence of the multichannel cochlear implant on vestibular function. Otolaryngol Head Neck Surg. 1998 Jun;118(6):900-2.

8. Kubo T, Yamamoto K, Iwaki T, Doi K, Tamura M. Different forms of dizziness occurring after cochlear implant. Eur Arch Otorhinolaryn- gol. 2001 Jan;258(1):9-12.

9. Todt I, Basta D, Ernst A. Does the surgical approach in cochlear implantation influence the occurrence of postoperative vertigo? Otolaryngol Head Neck Surg. 2008 Jan;138(1):8-12.

10. Tien HC, Linthicum FH Jr. Histopathologic changes in the vestibule after cochlear implantation. Otolaryngol Head Neck Surg. 2002 Oct; 127(4):260-4.

11. Handzel O, Burgess BJ, Nadol JB Jr. Histopathology of the peripheral vestibular system after cochlear implantation in the human. Otol Neurotol. 2006 Jan;27(1):57-64.

12. Nordfalk KF, Rasmussen K, Hopp E, Bunne M, Silvola JT, Jablonski GE. Insertion depth in cochlear implantation and outcome in residual hearing and vestibular function. Ear Hear. 2016 Mar-Apr;37(2): e129-37.

13. Nordfalk KF, Rasmussen K, Hopp E, Greisiger R, Jablonski GE. Scalar position in cochlear implant surgery and outcome in residual hearing and the vestibular system. Int JAudiol. 2014 Feb;53(2):121-7.

14. Xu XD, Zhang XT, Zhang Q, Hu J, Chen YF, Xu M. Ocular and cervical vestibular-evoked myogenic potentials in children with cochlear implant. Clin Neurophysiol. 2015 Aug;126(8):1624-31.

15. Frodlund J, Harder H, Maki-Torkko E, Ledin T. Vestibular function after cochlear implantation: a comparison of three types of electrodes. Otol Neurotol. 2016 Dec;37(10):1535-40.

16. Enticott JC, Eastwood HT, Briggs RJ, Dowell RC, O'Leary SJ. Methylprednisolone applied directly to the round window reduces dizziness after cochlear implantation: a randomized clinical trial. Audiol Neurootol. 2011;16(5):289-303.

17. Rah YC, Park JH, Park JH, Choi BY, Koo JW. Dizziness and vestibular function before and after cochlear implantation. Eur Arch Otorhinolaryngol. 2016 Nov;273(11):3615-21.

18. Basta D, Todt I, Goepel F, Ernst A. Loss of saccular function after cochlear implantation: the diagnostic impact of intracochlear electrically elicited vestibular evoked myogenic potentials. Audiol Neurootol. 2008;13(3):187-92.

19. Katsiari E, Balatsouras DG, Sengas J, Riga M, Korres GS, Xenelis J. Influence of cochlear implantation on the vestibular function. Eur Arch Otorhinolaryngol. 2013 Feb;270(2):489-95.

20. Meli A, Aud BM, Aud ST, Aud RG, Cristofari E. Vestibular function after cochlear implant surgery. Cochlear Implants Int. 2016 May; 17(3):151-7.

21. Rossi G, Solero P, Rolando M, Spadola Bisetti M.Vestibular function and cochlear implant. ORL J Otorhinolaryngol Relat Spec. 1998 Mar-Apr;60(2):85-7.

22. Kluenter HD, Lang-Roth R, Guntinas-Lichius O. Static and dynamic postural control before and after cochlear implantation in adult patients. Eur Arch Otorhinolaryngol. 2009 Oct;266(10):1521-5.

23. Krause E, Louza JP, Hempel JM,Wechtenbruch J, RaderT, Gurkov R. Effect of cochlear implantation on horizontal semicircular canal function. Eur Arch Otorhinolaryngol. 2009 Jun;266(6):811-7.

24. Robard L, Hitier M, Lebas C, Moreau S. Vestibular function and cochlear implant. Eur Arch Otorhinolaryngol. 2015 Mar;272(3):52330.

25. Louza J, Mertes L, Braun T, Gurkov R, Krause E. Influence of insertion depth in cochlear implantation on vertigo symptoms and vestibular function. Am J Otolaryngol. 2015 Mar-Apr;36(2):254-8. 